\title{
Investigation of a traveling wave thermoacoustic engine in a looped-tube
}

\author{
Petr Novotný1 ${ }^{1,2, a}$, Shu-Shen Hsu ${ }^{2}$, An-Bang Wang ${ }^{2}$, and Tomáš Vít ${ }^{1}$ \\ ${ }^{1}$ Technical University of Liberec, Department of Power Engineering Equipment, Studentská 2, 461 17, Czech Republic \\ ${ }^{2}$ National Taiwan University, Institute of Applied Mechanics, No.1, Sec. 4, Roosevelt Rd., Taipei City 106, Taiwan
}

\begin{abstract}
In the present paper, four configurations of a traveling wave thermoacoustic engine in a looped tube were investigated by means of theoretical calculations and experiments. The effect of natural heat convection on their functionality was observed. Acoustic intensity was measured using a dual two microphone method. The stack was designed with few times higher dimension of channels than thermal penetration depth, due to the stack should not be called regenerator. Pressure distribution in the resonator was measured, and a good agreement with theoretical calculations from DELTAEC has been demonstrated.
\end{abstract}

\section{Introduction}

In thermoacoustic devices, thermal energy is directly converted into acoustic wave energy and vice versa. This is caused by the heat interaction between a solid material and adjacent gas at the thermal penetration depth of the compressible oscillatory flow. Essential theoretical aspects of thermoacoustics have been provided by Rott [1], while further theoretical analyses and experimental investigations have been performed by Swift [2].

Besides their many advantages, which include no mechanical moving parts and environmentally friendly working fluids, thermoacoustic engines (TAE) are promising technologies for reusing waste heat, providing refrigeration or for producing micro-cooling devices [3].

Depending on the type of sound wave through the stack or regenerator, there are two types of TA devices, the standing-wave (SW) and traveling-wave (TW), respectively. Swift and other authors have tested various kinds of SW engines, but thermal energy conversion into acoustic work was at a lower thermal efficiency. SW TAE work on an irreversible thermodynamic cycle, and their thermal efficiency ratio of producing acoustic power and heat input has been limited to $20 \%$ so far.

When a traveling sound wave passes through the regenerator, the heat interaction between the gas and the solid material undergoes a Stirling-like thermodynamic cycle. Ceperley $[4,5]$ designed a device to test the TW principle, but without higher amplification of acoustic power due to the low acoustic impedance of the working gas, which caused large viscous loosening at high acoustic velocities. Backhaus [6] demonstrated an inexpensive TAE that employed the inherently efficient Stirling cycle, allowing it to achieve a thermal efficiency of $30 \%$, which is comparable to conventional internal combustion engines and piston Stirling engines.

\section{A looped-tube traveling wave TAE}

The simplest form of a traveling wave TA engine is the looped-tube design. This device is equipped with two heat exchangers, which produces a temperature gradient on the stack between them. The spontaneous gas oscillations of the traveling wave are amplified in the regenerator and loop and run around the loop through the regenerator from cold to hot.

There are two parameters that can affect TA energy conversion. One is $\omega \tau$, where $\omega$ is the angular frequency of gas oscillation, and $\tau$ is the time required for thermal equilibrium in the cross section of the channel. The time $\tau$ depends on the ratio of the characteristic transverse length of the pore (channel) to the thermal diffusivity of the working gas. If $\omega \tau \ll 1$, the gas in the channel moves in reverse and equilibrates at the local wall temperature. When $\omega \tau \gg 1$, the gas motion becomes isoentropic but still reversible to within a reasonable approximation. The oscillation gas becomes thermodynamically irreversible near $\omega \tau \sim 1$ due to incomplete heat transfer to the wall which is base for SW devices.

The small pore size maintains thermodynamically reversible heat transfer within the regenerator, so thermal resistivity is negligible. TWE also have an inherently higher efficiency than SWE. One considerable source of inefficiency is the viscous resistance in the regenerator. To minimize the impact of viscous resistance in the regenerator, TWE should be designed (inertance, compliance, and regenerator) so that $\left|P / U_{\mathrm{m}}\right| \gg \rho_{\mathrm{m}} c / A$; the acoustic impedance should be the highest at the cold end of the regenerator in order to avoid large viscous losses. Furthermore, the regenerator acts like an amplifier on volume velocity with a rough amplification of $T_{\mathrm{H}} / T_{\mathrm{C}}$.

\footnotetext{
${ }^{a}$ Corresponding author: petr.novotny@outlook.com
} 
The second parameter is the phase delay $\phi$ between the pressure $P=p e^{i \omega t}$ and the cross-sectional mean velocity, expressed as

$$
\begin{gathered}
U_{\mathrm{m}}=u_{\mathrm{m}} e^{i(\omega t+\phi)} \\
=u_{\mathrm{m}} \sin \phi e^{i(\omega t+\pi / 2)}+u_{\mathrm{m}} \cos \phi e^{i \omega \tau},
\end{gathered}
$$

where the first term is the standing wave component of $U_{\mathrm{m}}$, which is $\pi / 2$ out of phase with pressure $P$, and the second is the traveling wave component, which is in phase with $P$. Only the traveling part contributes to acoustic intensity $I$, and the acoustic energy is propagated in the same direction as the wave [7]. The time-averaged acoustic power of the wave as it passes through the regenerator can be expressed as

$$
\dot{W}=\frac{1}{2} \operatorname{Re}\left[P \widetilde{U}_{\mathrm{m}}\right]=\frac{1}{2}|\widetilde{P}|\left|\widetilde{U}_{\mathrm{m}}\right| \cos \phi,
$$

where $\operatorname{Re}[]$ and the tilde denote the real part and complex conjugate, respectively [9].

\section{Experimental setup}

In last experiment, the open-air, standing-wave thermoacoustic prime mover [8] was investigated. The effect of natural heat convection on the performance of the TW engine (not yet published) was observed. In the present study, the effect of the spatial orientation and position of the TA core in the loop-tube traveling-wave engine are discussed. Gathering more detailed knowledge of the acoustic wave and temperature in the loop-tube was also an objective of the experimental investigation and the subsequent theoretical calculation using the computer program DELTAEC.

\subsection{Thermoacoustic core}

The thermophysical properties of the working fluid, the design, and the dimensions of a TA device affect its operation. The straight part of the resonator with the TA core was the same as in the last experimental investigation into the SW prime mover. The dimension of the ceramic stack, with rectangular parallel channels, was also kept the same for the TW experiment. A stack length of $l_{\mathrm{S}}=50 \mathrm{~mm}$, with a cell density of $200 \mathrm{CPSI}$, consisting of rectangular pores with an inner size of $b \doteq 1.35 \mathrm{~mm}$ and a wall thickness of $s \doteq 0.4 \mathrm{~mm}$, produces a blockage ratio of 0.6 . The ratio of pore size and thermal penetration depth of $b / \delta_{\mathrm{k}} \doteq 7$ and for this reason the stack cannot be called regenerator.

The hot heat exchanger (HX) was made from resistance wire with a diameter of $0.45 \mathrm{~mm}$; heating power was kept at $52.4 \mathrm{~W}$ with a corresponding temperature of $294^{\circ} \mathrm{C}$ on the stack during main experiment.

The cooling heat exchanger (CX) was fabricated using three brass tubes with outer diameters of $6.35 \mathrm{~mm}$ and was hard-mounted in the body of the stainless steel part of the resonator at a distance of $200 \mathrm{~mm}$ from one end.

\subsection{Resonator and rig configurations}

The wavelength and frequency were the main parameters used to determine the total length of the device. The TW thermoacoustic device had a total length $L$ that was equivalent to the wavelength $L=\lambda=c / f$.

The resonator, with constant diameter of $60 \mathrm{~mm}$, had a total length of $1968 \mathrm{~mm}$, and it constituted a working frequency of approximately $f=180.5 \mathrm{~Hz}$. The loop-tube was made from a PVC tube, and the thermoacoustic core was assembled in four configurations due to the fixed position of the CX, as shown in Figure 1.

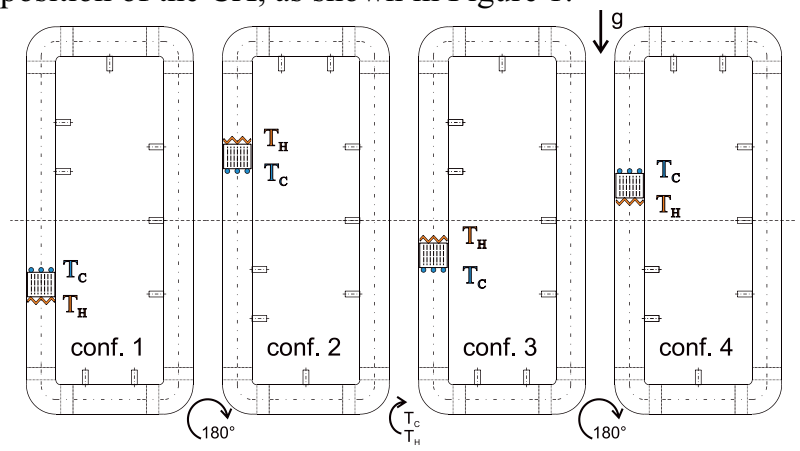

Figure 1. Four configurations of the TW-TAE in a looped tube

Each rig positions was tested with a maximum heating power of $70 \mathrm{~W}$ but only configuration No. 3 performed self-sustained acoustic oscillations. In this arrangement, natural heat convection had negative (HX above stack) for heat transfer into/from the stack with respect to gravity, but the temperature gradient $\nabla \mathrm{T}=\left(T_{\mathrm{H}}-T_{\mathrm{C}}\right) / l_{\mathrm{S}}$ could be maintained higher than in reversed configurations. According to the theory, the acoustic wave was propagated from cold to hot.

\subsection{Experimental procedure}

The temperatures of the stack were measured via thermocouples. The onset temperature difference for rig configuration No. 3 was determined at around $220^{\circ} \mathrm{C}$, at the lowest heating power of $45 \mathrm{~W}$. This is about $70^{\circ} \mathrm{C}$ higher than in an SW engine with a heating power under $20 \mathrm{~W}$.

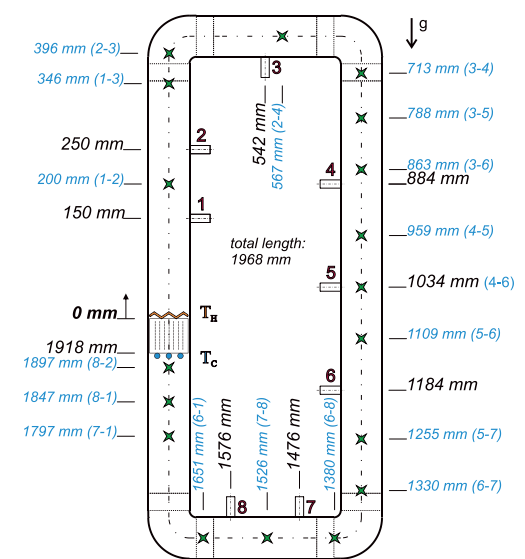

Figure 2. Measuring positions of microphones in the loopedtube of TW-TAE

Figure 2 shows the positions of the eight inputs into the loop-tube resonator, which were sealed or substituted 
with a pair of quarter-inch microphones or with a plug with thermocouple. The positions were measured from $\mathrm{HX}(0 \mathrm{~mm})$ in a clockwise direction.

Temperature distribution was measured for each position in steady-state with a constant heating power of $52.4 \mathrm{~W}$. An increase in temperature due to heat convection in the resonator above the $\mathrm{HX}\left(294^{\circ} \mathrm{C}\right)$ to 100 to $95^{\circ} \mathrm{C}$ in the first two and a temperature of $32{ }^{\circ} \mathrm{C}$ was observed in the third place on the top of rig. Placing an additional ambient heat exchanger above the HX should prevent this negative heat transfer.

The frequency, acoustic pressure amplitudes, and their phase shift were recorded by LabVIEW for selected pairs of microphones; these combinations are also depicted in Figure 2. The phase shift between two pressure transducers $(\Delta \mathrm{x})$ allowed the sound intensity at their middle point to be calculated. The simple form of the intensity equations of the two microphone method is given by [4]

$$
\begin{aligned}
I \cong \frac{1}{2 \omega \rho \Delta x}[(1- & \left.\frac{\delta_{v}}{r_{0}}\right)\left|p_{\mathrm{A}}\right|\left|p_{\mathrm{B}}\right| \sin \theta \\
& \left.+\frac{\delta_{v}}{2 r_{0}}\left(\left|p_{\mathrm{A}}\right|^{2}-\left|p_{\mathrm{B}}\right|^{2}\right)\right],
\end{aligned}
$$

where $\theta=\arg \left[p_{\mathrm{A}} / p_{\mathrm{B}}\right]$ represents the phase lead of $p_{\mathrm{A}}$ relative to $p_{\mathrm{B}}, r_{0}$ is the radius of the resonator with viscosity boundary layer $\delta_{v}=\sqrt{2 v / \omega}$, where $v$ stands for the kinematic viscosity of gas. The first terms in Eq. 3 are more significant for a traveling-wave form.

\section{Experimental results and discussion}

The temperature above HX has a greater influence on the pressure measurements in the first two positions. The data of these measurements have been analyzed with respect to temperature differences.

When the pressure oscillation reaches a stationary condition, the temperature in the regenerator (stack) usually also becomes stationary, yet we could still observe the influence of the negative heat transfer from the HX into the resonator. However, the frequency, pressure amplitudes, as well as phase between them, were recorded at steady-state, and the acoustic intensity in the middle point was determined by Eq. 3. The averaged RMS pressure in the resonator is shown in Figure 3.

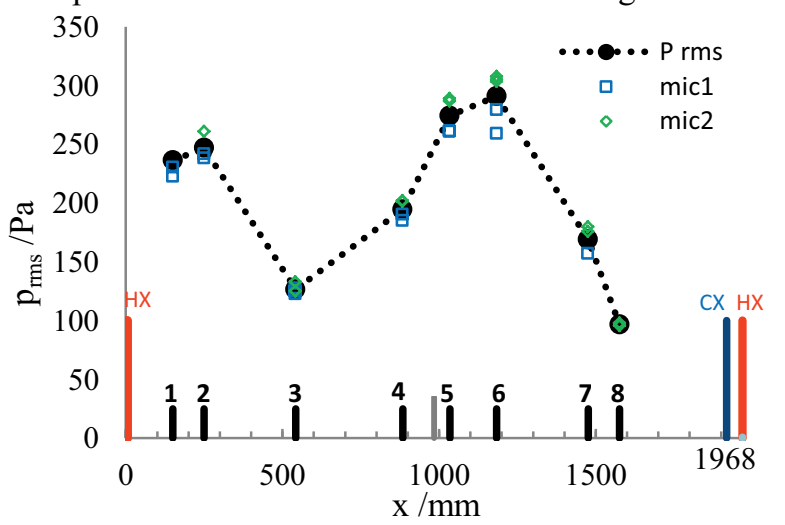

Figure 3. The distribution of RMS pressure $(\bullet)$ along the loop
We can see that maximum pressure ( $290 \mathrm{~Pa} \mathrm{rms}$, which corresponds with $820 \mathrm{~Pa}$ of peak-to-peak pressure) was detected at position 6 at $1184 \mathrm{~mm}$ from the HX. Knowing that the length of resonator was equaled to the one wavelength, then in the resonator existed two nodes and two antinodes of the pressure and also volume velocity. We also knew that the regenerator works as an acoustic power amplifier and that amplified acoustic power runs out from the HX. Essentially, the loop-tube is an acoustic waveguide, which provides feedback to $\mathrm{CX}$.

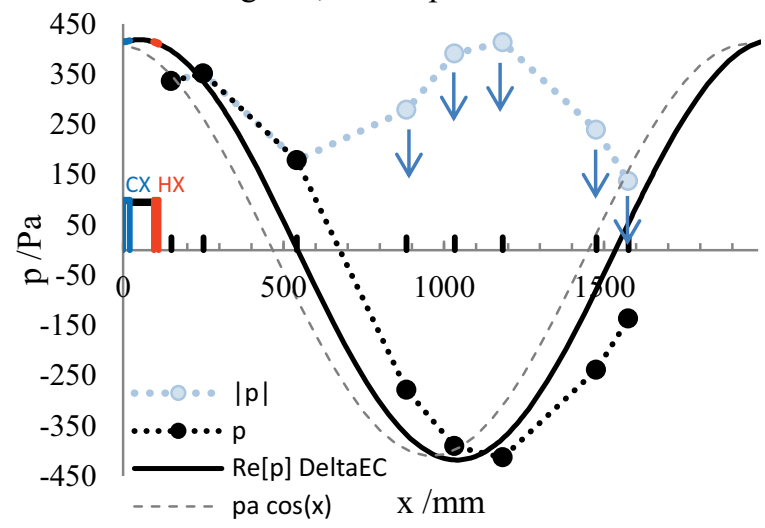

Figure 4. Distribution of acoustic pressure along the loopedtube according to measurements $(\bullet)$ and calculation $(-)$

Figure 4 shows acoustic pressure distribution in the looped-tube according to experimental data and theoretical calculations by DELTAEC [12]. A qualitative comparison exhibits good agreement. The pressure distribution in the simple loop-tube has roughly cosine curve. We can also observe two pressure nodes that should correspond with the antinode of an imaginary part of the volume velocity.

Acoustic intensity was measured using a two microphone method in selected coupled positions. The acoustic intensity should have been measured by the closest sensor; acoustic intensity differed greatly for microphones over a long distance when the phase shift was more than $90^{\circ}$.

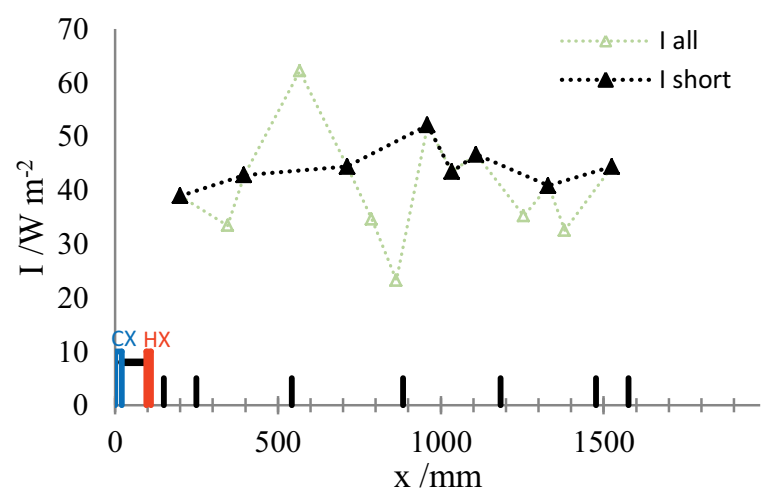

Figure 5. Distribution of acoustic intensity along the loopedtube for measurements with short $(\boldsymbol{\Delta})$ or long $(\triangle)$ distances

Figure 5 shows acoustic intensity in the resonator as established by a dual microphone method, using Eq. 3. It also depicts the ineffectual combination of two microphones at a greater distance and their deviations from the others. It was expected that acoustic intensity 
would exhibit a decreasing course in the loop-tube from the HX back to the CX (confirmed by DeltaEC), however this is not apparent from the experimental investigation. This could be the result of many factors, including the simplicity of Eq. 3. Further investigations should test using a different form of the two microphone method expression from [9]. However, for power measuring, it is necessary to measure pressure and velocity in an acoustic field or to use a two microphone method for its determination.

The measured resonance frequency was $180.5 \mathrm{~Hz}$, while the calculated resonance frequency was $175 \mathrm{~Hz}$, which was mainly the result of the non-uniform temperature of the working air in the loop-tube.

\section{Conclusions}

A traveling-wave thermoacoustic engine was designed, assembled, and tested in order to generate acoustic power in various configurations and angles with respect to the direction of gravity.

The acoustic pressure distribution in the looped-tube was measured and compared with theoretical calculations from DeltaEC and demonstrated good agreement. The intensity of the acoustic wave inside of the resonator was determined via a two microphone method. It was expected that the intensity of the acoustic wave would decrease in the direction from the stack back to the cold side due to viscous losses; however, the present results exhibited a different trend.

Higher efficiency cannot be achieved in the simple TW thermoacoustic prime in a looped-tube due to the low acoustic impedance of the working gas in the resonator. Not using so-called regenerator, the constant diameter of the resonator, and the spatial orientation and configuration of the TW TA engine have an apparent effect on its functionality.

The negative effect of natural heat convection was revealed when the heat was transported into the loopedtube. The use of a secondary ambient heat exchanger after the HX is a good solution for this problem and should be included in the engines.

In order for TA devices to achieve high performance and efficiency, it is necessary to use a combination of noble gases, to choose an adequate regenerator [10,11], and to determine the optimal compliance and feedback inertance of the resonator.

Further investigation will lead to constructing a thermoacoustic prime mover with higher efficiency, yet it is necessary to continue improving the performance of cold and hot heat exchangers and to choose a suitable regenerator.

The theoretical calculations provided by DeltaEC offer a good design guideline and will continue to be very helpful in the design and modifications of TW-TA engines in the future.

\section{Acknowledgments}

The authors are grateful for the financial support provided by the Grant Agency of the Czech Republic GACR under project No. P101/11/J019 and by the National Science Council in Taiwan under project No. NSC 100-2923-E-002-002-MY3. The authors also appreciate the support received through international cooperation in the research of thermoacoustics from The International Visegrad Fund-national Science Council, Taiwan Scholarship Program. The authors would also like to thank Dr. G. W. Swift for his valuable advice concerning DELTAEC code.

\section{References}

1. N. Rott, Advances in Applied Mechanics 20, 135 (1980)

2. G. W. Swift, Journal of the Acoustical Society of America 84, 1145 (1988)

3. O. G. Symko, E. A. Rahman, Y. S. Kwon, M. Emmi, R. Behunin, Microelectronics Journal 35, 185 (2004)

4. P. H. Ceperley Journal Acoustic Society of America 66, 1508 (1979)

5. P. H. Ceperley Journal Acoustic Society of America 77, 1508 (1985)

6. S. Backhaus, G. W. Swift, Nature 399, 335 (1999)

7. T. Yazaki, A. Iwata, T. Maekawa, and A. Tominaga, Physical Review Letters 81, 3128 (1998)

8. Y. Chen, A.Wang, in The International Conference on Green Technologies (2010)

9. A. M. Fusco, W. C. Ward, G. W. Swift, The Journal of the Acoustical Society of America 91, 2229 (1992)

10. A. S. Abduljalil, Z. Yu, A. J. Jaworski, International Journal of Engineering and Applied Sciences 5, 139 (2009)

11. A. S. Abduljalil, Z. Yu, A. J. Jaworski, Materials and Design 31, 217 (2011)

12. B. Ward, J. Clark, G. Swift, Design environment for low amplitude thermoacoustic engines (DeltaEC) User guide, (Los Alamos National Laboratory, 2008) 